\title{
Small or Non-Small Cell Lung Cancer Based Therapy for Treatment of Large Cell Neuroendocrine Cancer of The Lung? University of Cincinnati Experience
}

\author{
Ihab Eldessouki $\mathbb{D}^{1},{ }^{1}$ Ola Gaber $\mathbb{D}^{1},{ }^{1}$ Tariq Namad $\left(\mathbb{D},{ }^{1}\right.$ Jiang Wang, \\ John C. Morris $\mathbb{D}{ }^{1}$ and Nagla Abdel Karim $\mathbb{D}^{1}$ \\ ${ }^{1}$ Department of Hematology-Oncology, Vontz Center for Molecular Studies, University of Cincinnati, 3125 Eden Ave, Cincinnati, \\ $\mathrm{OH} 45267$, USA \\ ${ }^{2}$ Department of Pathology, University of Cincinnati, Laboratory Medicine Building, Suite 110, 234 Goodman Street, Cincinnati, \\ OH 45219-0533, USA
}

Correspondence should be addressed to Ihab Eldessouki; ihab_del@yahoo.com

Received 17 June 2018; Accepted 20 October 2018; Published 1 November 2018

Academic Editor: James L. Mulshine

Copyright (C) 2018 Ihab Eldessouki et al. This is an open access article distributed under the Creative Commons Attribution License, which permits unrestricted use, distribution, and reproduction in any medium, provided the original work is properly cited.

\begin{abstract}
Large cell neuroendocrine cancer (LCNEC) of the lung exhibits morphological and immunohistochemical characteristics of both neuroendocrine and large cell carcinomas. No defined optimal therapy has been described for this subset of patients and the question of whether these patients should be treated with non-small cell lung cancer (NSCLC) treatment protocols, according to the National Comprehensive Cancer Network (NCCN) guidelines, or with small cell lung cancer (SCLC) due to histological and clinical similarities is still uncertain. We conducted a retrospective review of patients identified with diagnosis of LCNEC of the lung at the University of Cincinnati Cancer Center from the year 2002 to 2012 to determine which treatment approach resulted in improved outcomes in this rare category of disease. Patients who received chemotherapy whether NSCLC (group A) or SCLC (group B) protocols did not show significant changes in OS $(\mathrm{P}=0.911)$. Meanwhile, patients who underwent surgery (group C) had better OS compared to groups A and $\mathrm{B}(\mathrm{P}=0.027$ and 0.024 , respectively). This analysis reveals that outcomes for SCLC or NSCLC treatment strategies in LCNEC patients did not result in survival advantages and future research should be addressing it as a separate entity.
\end{abstract}

\section{Background}

Clinically, LCNEC of the lung resembles SCLC rather than carcinoid tumors. It is characterized by early nodal and distant metastatic spread [1], and presenting symptoms include cough, hemoptysis, chest pain, dyspnea, and weight loss and this mimics other NSCLC and SCLC clinical presentations; also, paraneoplastic syndromes are considered uncommon in LCNEC [2]. These patients have poor prognosis, and the natural history of the disease closely mimics that of SCLC $[1,3]$. Molecular profiling reveals heterogeneous pattern that reveals characteristics of both SCLC and NSCLC, with close resemblance to adenocarcinoma. Rekhtman et al. reported results from 45 resected LCNEC tumors which underwent targeted next-generation sequencing of 241 genes [4]. Fifty-six percent of the tumors had NSCLC-like molecular features, such as lack of RB1+TP53 coalteration and presence of NSCLC-type common mutations including STK11, KRAS, KEAP1, and NFE2L2, while 40\% showed SCLC-like molecular features, with $R B 1+T P 53$ coalteration, complete absence of mutations in STK11 and KRAS, and exclusive or enriched occurrence of MYCL, SOX2, and FGFR1 amplifications and mutation and/or loss of PTEN [4]. So far, no standard treatment approach for LCNEC has been defined and existing recommendations are extrapolation of NSCLC and SCLC therapies. The reasons may include rarity of the disease, but the lack of randomized prospective trials is a major factor. [3]. According to the NCCN guidelines, LCNEC can be managed as per NSCLC guidelines; however, others prefer 
same chemotherapeutic regimens for SCLC, based on the fact that both are high-grade neuroendocrine tumors and due to their similar clinical course [5]. Even though resection is recommended for early stage LCNEC [6], the expected prognosis remains poor with a 5-year survival rate of 27 to $67 \%$ [7]. The role of adjuvant chemotherapy is only supported by data obtained from small prospective phase II studies [8] and retrospective experiences with adjuvant treatment $[9,10]$. SCLC regimens (platinum + etoposide) are suggested to be more effective and beneficial than those of NSCLC [11].

Another debatable aspect in LCNEC management is radiotherapy and prophylactic cranial irradiation (PCI) in early stages. Despite the poorer prognosis and short survival, available evidence supports administration of radiotherapy according to NSCLC guidelines. The low incidence of spontaneous brain metastases (about 25\%) does not support routine PCI as in SCLC [12].

In advanced stage LCNEC, SCLC regimens more commonly practiced which is based on limited retrospective analyses [13] and according to the recommendations of American Society of Clinical Oncology (ASCO) in 2015 [14]. Recently, it was suggested that there is an increase in the OS in LCNEC patients with NSCLC regimens which are adopted, specially gemcitabine-platinum rather than pemetrexed-platinum and etoposide-platinum (SCLC-based) regimens [15]. In this study, we are reporting the results of a retrospective analysis of LCNEC patients treated in the University of Cincinnati medical center from 2002 to 2012. Our aim was to see how the treatment approach affected survival, responses, and prognosis of pulmonary LCNEC.

\section{Methods}

A retrospective review of patients identified with a pathological diagnosis of LCNEC of the lung. Database of Medical Center of the University of Cincinnati was searched from the year 2002 to 2012, and all patients with LCNEC histology were identified. Patients with incomplete records were removed from the study.

Paraffin blocks were retrieved from the tissue bank for the eligible subjects and their diagnosis was reviewed according to WHO 2015 guidelines. Tissue markers tested included CD56, synaptophysin, chromogranin A, and Ki-67 [2, 16-18].

Survival probabilities were estimated by Kaplan Meier method and differences in survival were compared by the log-rank test. Uni- and multivariable predictors of overall mortality were estimated by Cox-regression analysis. Overall survival was defined as the time from diagnosis till the time of death from any cause.

\section{Results}

3.1. Demographics. Searching the database of the Cancer Center of the University of Cincinnati, we were able to identify 26 patients diagnosed as LCNEC of the lung. The median age of the patients was 58 years (range: $42-77$ years), and males to females ratio was 1.2 . Six patients $(23.1 \%)$ were nonsmokers, and 14 patients (53.8\%) were heavy smokers. For patients' demographics, refer to Table 1.

3.2. Patient Management. Nine patients (34.6\%) were treated as SCLC patients, i.e., received cisplatin/etoposide for their initial treatment, and 6 patients (23.1\%) received a NSCLCbased treatment. The median OS for all patients was 16 months (range: 11 to 42 months), and OS of patients treated with chemotherapy was found to be statistically insignificant compared to those who never received chemotherapy (HR 0.43 and $0.79 ; p=0.23$ and 0.71 ). Patients who underwent surgery had a higher risk of death (HR of 3.66; $\mathrm{p}=0.04$ ).

3.3. Mortality Hazard and Survival Analysis. In the univariate model analysis, increasing age was found to be statistically significant (HR: 0.95, 95\% CI: 0.90-0.99, P = 0.032), and group $\mathrm{C}$ patients never received chemotherapy during their course of treatment, and surgery was their main modality of treatment (HR 0.26, 95\% CI: 0.08-0.87, P = 0.029). On multivariable analysis (MVA), group $\mathrm{C}$ was associated with the highest statistical significance (HR: 0.30, 95\% CI: 0.05$1.81, \mathrm{P}=0.190)$. The stage III/IV disease overall mortality was higher than stages I/II; however this did not represent statistical significance (HR:2.26, $\mathrm{P}=0.128$ ); see Table 2 .

3.4. Kaplan-Meier Survival Analysis. Median survival (95\% CI) for each treatment group of A, B, and C was 2.75 (0.05$5.45), 2.88$ (1.46-4.30), and 9.87 years (2.56-17.18), respectively. Group C patients had the best overall survival (OS) rate [5year OS of $65.6 \%$ and median survival of 9.87 years $(95 \%$ CI: 2.56-17.18)], compared to those who received group A chemotherapy regimen [5-year OS of $30.5 \%$ and median survival of 2.75 years (95\% CI: $0.05-5.45$ )] or to those who received group B chemotherapy regimen [5-year OS of $22.2 \%$ and median survival of 2.88 years (95\% CI: $1.46-$ 4.30)][Figure 1].

\section{Discussion}

Molecular and genetic analysis of lung cancers have enabled defining subcategories that are now the foundation for targeted and immunotherapy. However, rare and ambiguous subtypes such as LCNEC of the lung and primary pulmonary sarcomatoid carcinoma (PPSC) are underpresented, and their treatment strategies are still based on more common lung tumor histologies such as NSCLC though they represent clinically and histologically independent entities [19-21]. In a study performed on 45 LCNEC patients by Sun et al., the authors reported that the response rates were $73 \%$ and $50 \%$ for SCLC and NSCLC treatment-based groups $(P=0.19)$, respectively. The median progression free survival was higher for the SCLC group (6.1 versus 4.9 months, $\mathrm{P}=0.41$ ), and the OS showed that SCLC group was also at advantage (16.5 versus 9.2 months, $\mathrm{P}=0.10$ ) compared to NSCLC-based treated group [13]. Our results showed that the NSCLC group did not show a survival advantage compared to the SCLC group. Although NSCLC group had slightly higher median OS ( 0.05 years) than SCLC treatment-based group, the 5-year 


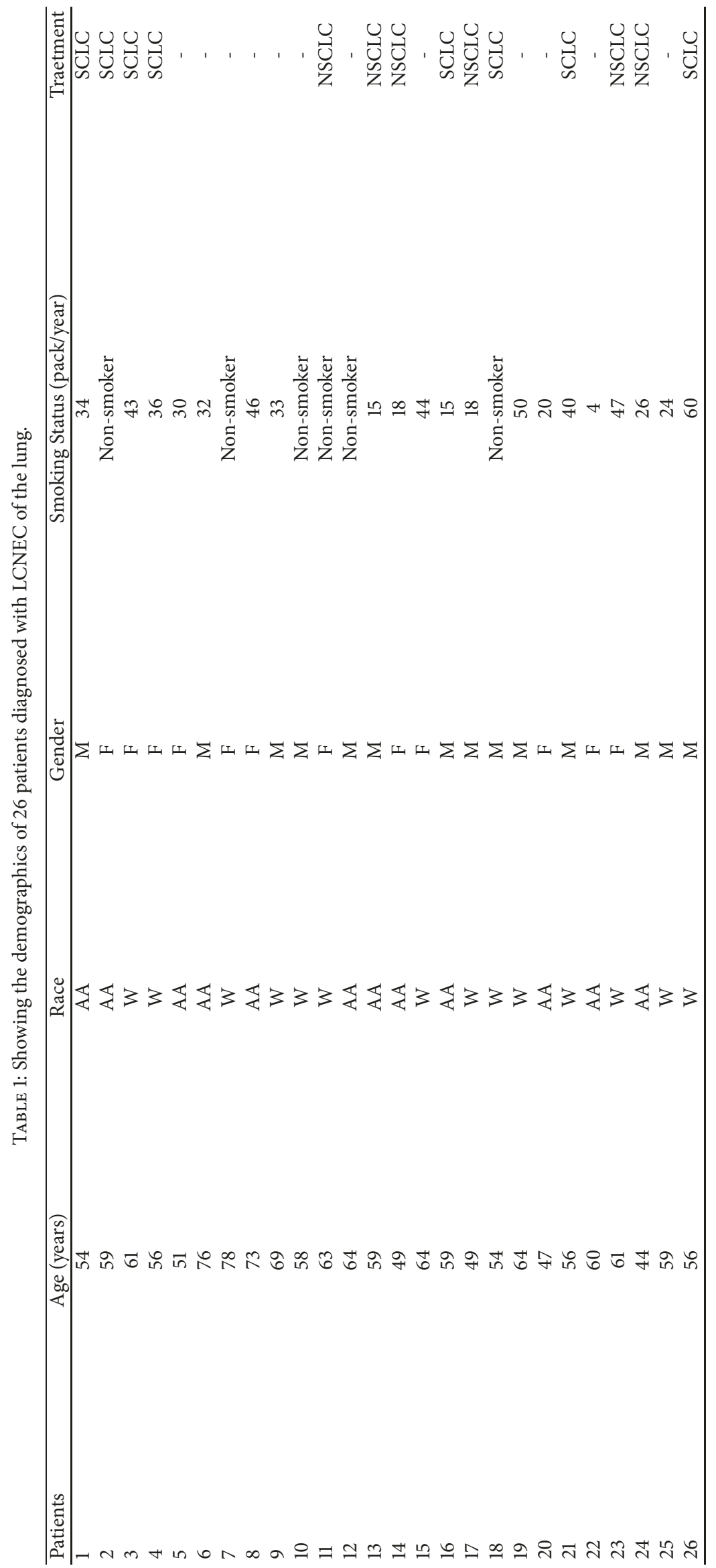




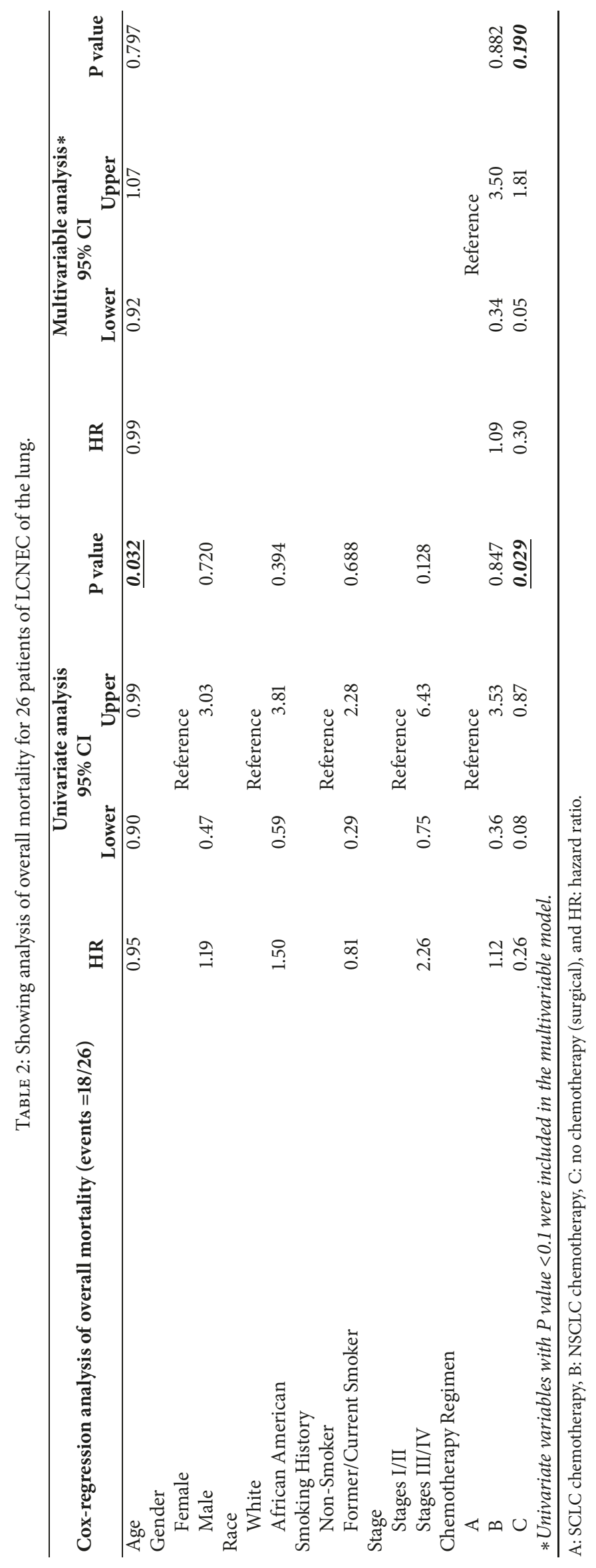




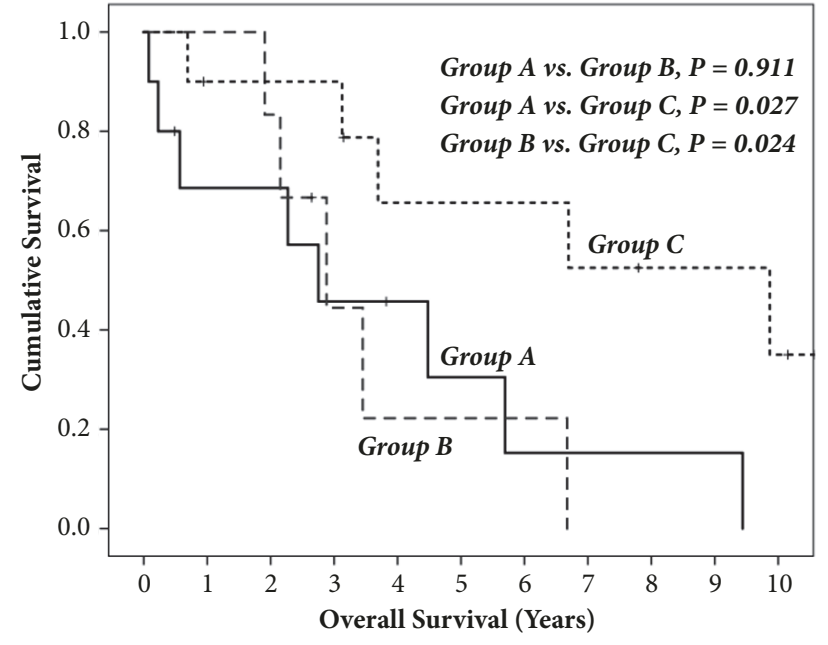

FIGURE 1: The figure compares the OS in years against the cumulative survival of each treatment group. Groups A and B compared did not have statistical significance $(\mathrm{p}=0.911)$. Comparing groups $A$ and $B$ to group $\mathrm{C}$, the latter exhibits significance in survival with both groups ( $\mathrm{p}=0.027$ and 0.024 , respectively).

OS was lower in comparison; however, there was no statistical significance in both results $(\mathrm{p}=0.911)$. LCNEC of the lung prognostic factors was reported to be similar to SCLC rather than carcinoid tumors [1]. In a study comparing the 5-year survival of stage I LCNEC and poorly differentiated NSCLC patients, significant higher survival rates were revealed in the poorly differentiated NSCLC patients (67\% versus $88 \%$, $\mathrm{p}=003$ ) [22], indicating that poor effect of currently executed regimens for these rare but rather specific categories. A retrospective analysis revealed that LCNEC 5-year survival rates range from 15 to $57 \%$, and diagnosis at an early stage showed slight improvement in these cases (27-67 \%) [23], regardless of treatment strategy. Stage to stage 5-year survival comparison of pulmonary LCNEC and NSCLC was 54.5\% versus $89.3 \%$ for the adenocarcinoma or squamous cell carcinoma patients [24]. It is common for LCNEC of the lung to metastasize to distant sites [22], and adjuvant treatment was suggested in most platforms [13, 23, 25, 26]. However reports were more in favor of platinum based therapies with unsatisfactory outcomes (recurrence rate $=46 \%$ ) [22], even though platinum based regimens yielded higher response rates compared to NSCLC [13].

\section{Conclusions}

LCNEC of the lung has poor prognosis and survival. Studies report inconsistencies in the results from these patients' treatment. Even though group $\mathrm{C}$ patients, who were treated with surgery only, had better survival, recurrences were common and survival was poor. More randomized trials are needed to address this category of tumors probably based on tumor specific targeted treatment. Programmed cell death 1 (PD-1)/programmed cell death ligand 1 (PD-L1) expression, immunotherapy, and their outcomes in LCNEC open a new door for considering a new standard treatment [27-31].

\section{Data Availability}

The data used to support the findings of this study are included within the article.

\section{Conflicts of Interest}

The authors declare that there are no conflicts of interest.

\section{Authors' Contributions}

Ihab Eldessouki was responsible for statistics and writing. Ola Gaber was responsible for writing. Tariq Namad and Mohamad Sakkal were responsible for data collection. Jiang Wang was responsible for pathological review and data collection. John C. Morris was responsible for scientific rational and patient management. Nagla Abdel Karim was responsible for final supervision and patient management.

\section{References}

[1] H. Asamura, T. Kameya, Y. Matsuno et al., "Neuroendocrine neoplasms of the lung: A prognostic spectrum," Journal of Clinical Oncology, vol. 24, no. 1, pp. 70-76, 2006.

[2] M. Fasano, C. M. Della Corte, F. Papaccio, F. Ciardiello, and F. Morgillo, "Pulmonary Large-Cell Neuroendocrine Carcinoma: From Epidemiology to Therapy," Journal of Thoracic Oncology: Official Publication of The International Association for The Study of Lung Cancer, vol. 10, no. 8, pp. 1133-4111, 2015.

[3] B. S. Glisson and C. A. Moran, "Large-cell neuroendocrine carcinoma: Controversies in diagnosis and treatment," JNCCN - Journal of the National Comprehensive Cancer Network, vol. 9, no. 10, pp. 1122-1129, 2011.

[4] N. Rekhtman, M. C. Pietanza, M. D. Hellmann et al., "Nextgeneration sequencing of pulmonary large cell neuroendocrine carcinoma reveals small cell carcinoma-like and non-small cell carcinoma-like subsets," Clinical Cancer Research, vol. 22, no. 14, pp. 3618-3629, 2016.

[5] J. Naidoo, M. L. Santos-Zabala, T. Iyriboz et al., "Large Cell Neuroendocrine Carcinoma of the Lung: Clinico-Pathologic Features, Treatment, and Outcomes," Clinical Lung Cancer, vol. 17, no. 5, pp. e121-e129, 2016.

[6] J. Zacharias, A. G. Nicholson, G. P. Ladas, and P. Goldstraw, "Large cell neuroendocrine carcinoma and large cell carcinomas with neuroendocrine morphology of the lung: Prognosis after complete resection and systematic nodal dissection," The Annals of Thoracic Surgery, vol. 75, no. 2, pp. 348-352, 2003.

[7] G. Lo Russo, S. Pusceddu, C. Proto et al., "Treatment of lung large cell neuroendocrine carcinoma," Tumor Biology, vol. 37, no. 6, pp. 7047-7057, 2016.

[8] A. Iyoda, K. Hiroshima, Y. Moriya et al., "Prospective Study of Adjuvant Chemotherapy for Pulmonary Large Cell Neuroendocrine Carcinoma," The Annals of Thoracic Surgery, vol. 82, no. 5, pp. 1802-1807, 2006.

[9] A. Iyoda, K. Hiroshima, Y. Moriya et al., "Postoperative recurrence and the role of adjuvant chemotherapy in patients with pulmonary large-cell neuroendocrine carcinoma," The Journal of Thoracic and Cardiovascular Surgery, vol. 138, no. 2, pp. 446453, 2009.

[10] I. S. Sarkaria, A. Iyoda, M. Soo Roh et al., "Neoadjuvant and Adjuvant Chemotherapy in Resected Pulmonary Large Cell 
Neuroendocrine Carcinomas: A Single Institution Experience," The Annals of Thoracic Surgery, vol. 29, no. 4, pp. 1180-1187, 2011.

[11] G. Rossi, A. Cavazza, A. Marchioni et al., "Role of chemotherapy and the receptor tyrosine kinases KIT, PDGFR $\alpha, \operatorname{PDGFR} \beta$, and Met in large-cell neuroendocrine carcinoma of the lung," Journal of Clinical Oncology, vol. 23, no. 34, pp. 8774-8785, 2005.

[12] J. Rieber, J. Schmitt, A. Warth et al., "Outcome and prognostic factors of multimodal therapy for pulmonary largecell neuroendocrine carcinomas," European Journal of Medical Research, vol. 20, no. 1, 2015.

[13] J.-M. Sun, M.-J. Ahn, J. S. Ahn et al., "Chemotherapy for pulmonary large cell neuroendocrine carcinoma: Similar to that for small cell lung cancer or non-small cell lung cancer?" Lung Cancer, vol. 77, no. 2, pp. 365-370, 2012.

[14] G. A. Masters, S. Temin, C. G. Azzoli et al., "Systemic therapy for stage IV non-small-cell lung cancer: American society of clinical oncology clinical practice guideline update," Journal of Clinical Oncology, vol. 33, no. 30, pp. 3488-3515, 2015.

[15] J. L. Derks, R. J. van Suylen, E. Thunnissen et al., "Chemotherapy for pulmonary large cell neuroendocrine carcinomas: does the regimen matter?" European Respiratory Journal, vol. 49, no. 6, p. $1601838,2017$.

[16] G. Pelosi, A. Sonzogni, S. Harari et al., "Classification of pulmonary neuroendocrine tumors: New insights," Translational Lung Cancer Research, vol. 6, no. 5, pp. 513-529, 2017.

[17] A. Fisseler-Eckhoff and M. Demes, "Neuroendocrine tumors of the lung," Cancers, 2012.

[18] E. Thunnissen, B. I. Witte, M. Noguchi, and Y. Yatabe, "Reply to Letter "The Use of Immunohistochemistry Improves the Diagnosis of Small Cell Lung Cancer and Its Differential Diagnosis. An International Reproducibility Study in a Demanding Set of Cases."," Journal of Thoracic Oncology, vol. 12, no. 6, pp. e70-e71, 2017.

[19] N. A. Karim, J. Schuster, I. Eldessouki et al., "Pulmonary sarcomatoid carcinoma: University of Cincinnati experience," Oncotarget, vol. 9, no. 3, pp. 4102-4108, 2018.

[20] N. Karim, I. Eldessouki, M. Yellu, T. Namad, J. Wang, and O. Gaber, "A case study in advanced lung cancer patients with vimentin over expression," Clinical Laboratory, vol. 63, no. 10, pp. 10-7754, 2017.

[21] I. Eldessouki, O. Gaber, K. Riaz Muhammad, W. Jiang, and A. K. N. Wang Jiang, "Clinical Presentation and Treatment Options for Clear Cell Lung Cancer: University of Cincinnati A Case Series and Literature Review of Clear Cell Lung Cancer," Asian Pacific Journal of Cancer Prevention, Asian Pacific Organization for Cancer Prevention, 2000.

[22] H. Takei, H. Asamura, A. Maeshima et al., "Large cell neuroendocrine carcinoma of the lung: A clinicopathologic study of eighty-seven cases," The Journal of Thoracic and Cardiovascular Surgery, vol. 124, no. 2, pp. 285-292, 2002.

[23] A. Iyoda, T. Makino, S. Koezuka, H. Otsuka, and Y. Hata, "Treatment options for patients with large cell neuroendocrine carcinoma of the lung. General Thoracic and Cardiovascular Surgery," in June). Treatment options for patients with large cell neuroendocrine carcinoma of the lung, pp. 10-1007, Springer, General Thoracic and Cardiovascular Surgery, 2014.

[24] A. Iyoda, K. Hiroshima, Y. Moriya et al., "Prognostic impact of large cell neuroendocrine histology in patients with pathologic stage Ia pulmonary non-small cell carcinoma," The Journal of Thoracic and Cardiovascular Surgery, vol. 132, no. 2, pp. 312-315, 2006.
[25] A. Iyoda, K. Hiroshima, Y. Nakatani, and T. Fujisawa, "Pulmonary Large Cell Neuroendocrine Carcinoma: Its Place in the Spectrum of Pulmonary Carcinoma ${ }^{* *}$ A part of this report was presented by Iyoda A et al. Pulmonary large cell neuroendocrine carcinoma. JJLC 2006;46:315-20 [in Japanese with English abstract]." The Annals of Thoracic Surgery, vol. 84, no. 2, pp. 702-707, 2007.

[26] F. G. Fernandez and R. J. Battafarano, "Large-Cell Neuroendocrine Carcinoma of the Lung," Cancer Control, vol. 13, no. 4, Article ID 107327480601300404, pp. 270-275, 2006.

[27] A. Naik, M. Rahouma, I. Eldesoki et al., “50 Gene Panel Testing in Non-Small Cell Lung Cancer and Options for Targeted Therapy," CPQ Cancer, vol. 1, 2018, https://www.cientperiodique .com/article/CPQCR-1-2-10.pdf.

[28] N. Abdel Karim, A. Sendilnathan, I. Eldessouki et al., "PS06.06 Immune Checkpoint Markers in Lung Large Cell Neuroendocrine Carcinomas (L- LCNEC)," Journal of Thoracic Oncology, vol. 12, no. 11, pp. S1583-S1584, 2017.

[29] N. Abdel Karim, A. Sendilnathan, I. Eldessouki et al., "PS06.06 Immune Checkpoint Markers in Lung Large Cell Neuroendocrine Carcinomas (L- LCNEC)," Journal of Thoracic Oncology, vol. 12, no. 11, pp. S1583-S1584, 2017.

[30] N. Karim, J. Pathrose, and I. El Desouki, "P3.12-05 The Pattern of PD-L1 Expression in Thoracic Neuroendocrine Tumors," Journal of Thoracic Oncology, vol. 13, no. 10, pp. S971-S972, 2018.

[31] N. Karim, J. Pathrose, H. Fathallah et al., "P2.01-129 Potential Impact of KRAS Molecular Profiling of Non-Squamous NonSmall Cell Lung Cancer (NSCLC).," Journal of Thoracic Oncology, vol. 13, no. 10, pp. S714-S715, 2018. 


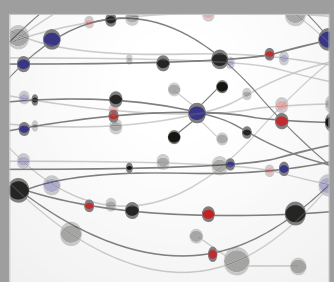

The Scientific World Journal
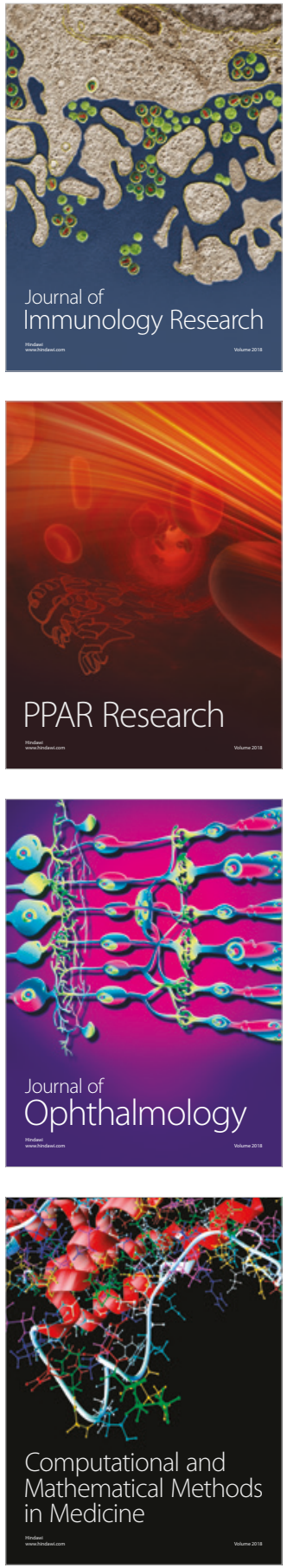

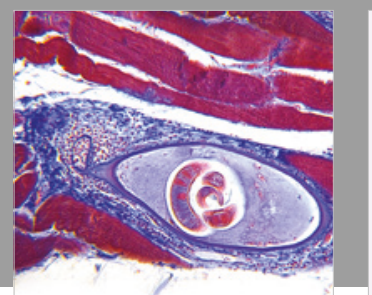

Gastroenterology Research and Practice

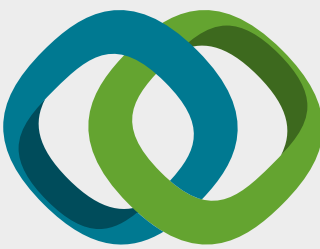

\section{Hindawi}

Submit your manuscripts at

www.hindawi.com
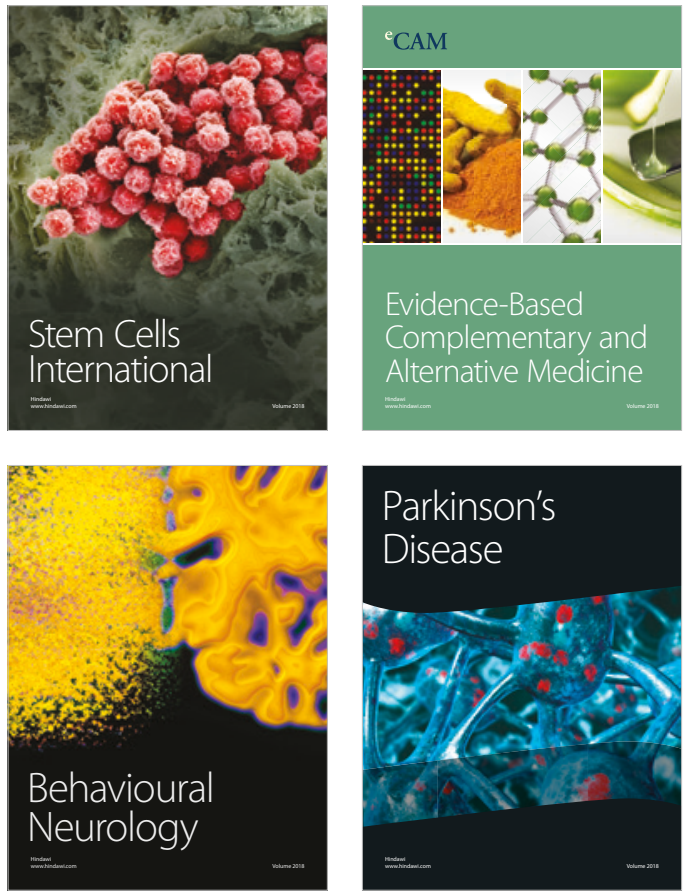

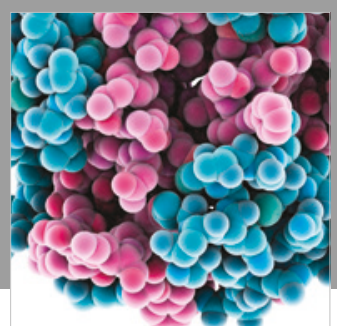

ournal of

Diabetes Research

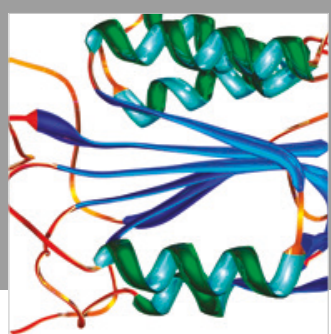

Disease Markers
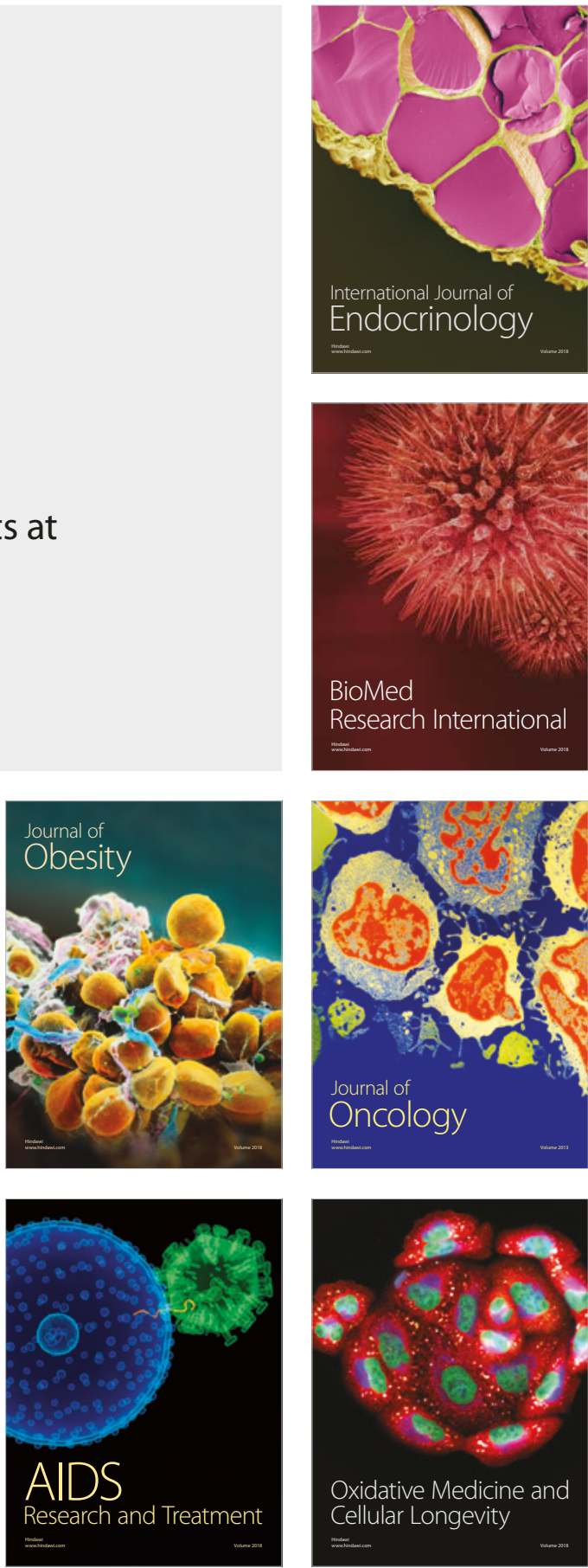